\title{
Atención de enfermería en la unidad de hemodiálisis a un paciente cada vez más anciano
}

\author{
Raquel Rodríguez Rivas, Alicia Sánchez García
}

Hospital Universitario Puerta de Hierro Majadahonda. Madrid

\section{Introducción:}

Los cuidados del paciente con enfermedad renal son de vital importancia en el desarrollo de su enfermedad. El aumento de la esperanza de vida en nuestro país y los avances científicos y tecnológicos, ponen en evidencia un importante aumento de la edad media de los pacientes con enfermedad renal crónica avanzada (ERCA) que reciben tratamiento renal sustitutivo (TRS), elevando su comorbilidad y grado de dependencia. Según datos del Instituto Nacional de Estadística (INE) nuestra estructura demográfica se enfrenta a un proceso continuo de envejecimiento que se ve acelerado por el descenso de la natalidad y los saldos migratorios negativos. Los mayores crecimientos de población se concentrarían en las edades avanzadas. Concretamente en 2052 el grupo de edad de mayores de 64 años se incrementaría en 8.2 millones de personas (un $89 \%$ ) y pasaría a constituir el $37 \%$ de la población total en España. Debido a la entrada en programa de hemodiálisis de pacientes de edad avanzada, nuestros cuidados se han visto modificados para poder adaptarse a sus necesidades. Esto conlleva un aumento de las cargas de trabajo en el personal de enfermería.

\section{Objetivos:}

El objetivo principal de nuestro estudio es conocer los grados de incapacidad física de nuestros pacientes. Los objetivos secundarios son identificar las cargas añadidas al personal de enfermería y buscar estrategias de actuación.

\section{Material y métodos:}

Se ha realizado una recogida de datos durante el mes de Abril de 2013 a todos los pacientes en activo en la unidad de diálisis hospitalaria. Las variables analizadas han sido las siguientes: edad, sexo, acceso vascular, inclusión en lista de trasplante y grado de incapacidad física. Se expresan en medias y desviación estándar. Análisis estadístico realizado con SPSS v15.0.

\section{Resultados:}

Se recogen datos de 80 pacientes, con una edad media de 64.8 años (DE 13.9) con un rango de 28 a 91 años; el $28.8 \%$ tienen 75 años o más. El $57.5 \%$ son varones. En cuanto al acceso vascular, el $47.5 \%$ son portadores de catéteres permanentes, el $43.8 \%$ tienen FAVI (fístulas autólogas y protésicas), y el $8.8 \%$ restante son portadores tanto de FAVI como catéter. En lista de espera para trasplante hay solo 7 pacientes $(8.8 \%)$, con una media de 52.9 años (DE 7.0). Para establecer los grados de incapacidad física, nos hemos basado en el índice global desarrollado por la Cruz Roja. El $16.3 \%$ de los pacientes se encuentran en los grados $4-5$, el $30 \%$ en los grados $2-3$ y el $53.8 \%$ en los grados $0-1$.

\section{Conclusiones:}

La entrada de pacientes de edad avanzada en programa de hemodiálisis implica un cambio en el trabajo diario del personal de enfermería. Con los cambios en las características de la población, debemos adecuarnos a las nuevas particularidades del enfermo renal de manera progresiva e individuali- 
zada, encaminándonos hacia la aplicación de unos cuidados integrales y no centrarnos sólo en los aspectos técnicos de nuestro trabajo.

\section{Referencias Bibliográficas}

1. Aladrén M.J., Pérez y Pérez J., Azuara M., Berisa F. Hemodiálisis en pacientes de edad avanzada. Estudio multicéntrico de las sociedades aragonesa y norte de nefrología. Nefrología 1999;19(1):3948.
2. M. De Francisco A.L., San Juan F., Foraster A., Fabado S., Carretero D., Santamaría C. Et al. Estudio epidemiológico de pacientes ancianos con insuficiencia renal crónica en hemodiálisis. nefrología 2008;28(1):48-55.

3. Pérez García R., Pacientes geriátricos en hemodiálisis. Diálisis en el anciano. Revista SEDEN 2001;4(·):64-73.

4. Nota de prensa del Instituto Nacional de Estadística (19 noviembe 2012) Disponible en www.ine.es/ prensa/np744.pdf. 